\title{
Superconductivity, Weak Itinerant Ferromagnetism and Electronic Band Structure of $\mathrm{Y}_{9} \mathrm{Co}_{7}$
}

\author{
A. KoŁodziejczyK ${ }^{a, *}$, B. Wiendlocha ${ }^{b}$, R. ZAlecki ${ }^{a}$ \\ J. TOBOŁA ${ }^{b}$ AND S. KAPRZYK ${ }^{b}$ \\ ${ }^{a}$ Department of Solid State Physics \\ ${ }^{b}$ Department of Condensed Matter Physics \\ AGH University of Science and Technology \\ al. Mickiewicza 30, 30-059 Cracow, Poland
}

The paper presents experimental results for the intermetallic compound $\mathrm{Y}_{9} \mathrm{Co}_{7}$ which is the first very weak itinerant ferromagnetic superconductor. The measurements of electrical resistivity, susceptibility, magnetization, specific heat, nuclear magnetic resonance, and other properties show the magnetic transition at $T_{\mathrm{C}} \approx 4.5 \mathrm{~K}$ and the onset of superconductivity at about $T_{\mathrm{S}} \approx 2.5 \mathrm{~K}$, revealing the coexistence state within a temperature interval below $T_{\mathrm{S}}$. A few of the results are shortly reviewed and discussed. We focus attention on our recent ultraviolet photoemission spectroscopy and their comparison with our band structure calculations. Interpretations are considered which take into account the characteristic structure of the compound and different possible types of magnetic ordering.

PACS numbers: 74.25.Jb, 74.25.Ha, 74.20.Mn

\section{Introduction}

The problem of the magnetism in Y-Co system was revived at the beginning of the eighties and much more interest was arisen since the discovery of superconductivity in $\mathrm{Y}_{4} \mathrm{Co}_{3}$ with the transition temperature $T_{\mathrm{S}} \approx 2 \mathrm{~K}$ [1], later found to be $\mathrm{Y}_{9} \mathrm{Co}_{7}[2]$. The intermetallic compound $\mathrm{Y}_{9} \mathrm{Co}_{7}$ is the first ever observed example of the coexistence of well experimentally characterized conventional low-temperature superconductivity $[1-8]$ and very weak itinerant ferromagnetism (VWIF) with the Curie temperature $T_{\mathrm{C}} \approx 4.5 \mathrm{~K}$ [1, 4, 9-19]. It shows very interesting and unique properties. In the paper [1] we observed for the first time in a transition metal alloy the presence of magnetic ordering below $T_{\mathrm{C}}$ and superconductivity below $T_{\mathrm{S}}$. It was found $[1,4,9-11,15-19]$ that in spite of previous thought [20-23] there exist

*corresponding author; e-mail: akolo@uci.agh.edu.pl 
itinerant magnetic moments on some cobalt positions and more local magnetic moments on the other cobalt positions in contrast to a prediction that $\mathrm{Y}_{9} \mathrm{Co}_{7}$ should be a Pauli paramagnet. A lot of work was done since that time up to about late eighties (for a review see, e.g. [4, 12-14]), but special features and the electronic structure of $\mathrm{Y}_{9} \mathrm{Co}_{7}$ are of particular interest and still remain to be explained.

The story of coexisting magnetism and superconductivity is a complex one, containing features associated with coexisting antiferromagnetically ordered phases, ferromagnetically ordered rare-earth systems (e.g. the rare-earth rhodium borides and the Chevrel phases), random solid solutions, where spin-glass freezing occurs, magnetically distinct bands of electrons that mediate magnetic interactions or become superconductive (e.g. heavy-fermion superconductors) [24, 25]. The interesting question arises: Do superconductivity and long-range magnetic order coexist in itinerant ferromagnets? Only recently it has been demonstrated that, in fact, such a coexistence occurs in $\mathrm{UGe}_{2}$ [26], $\mathrm{ZrZn}_{2}$ [27], and URhGe [28]. It is worth stressing that in one among our papers from 1984 [10] we suggested that such phenomenon might occur in $\mathrm{ZrZn}_{2}, \mathrm{InSc}_{3}, \mathrm{TiBe}_{1.8} \mathrm{Cu}_{0.2}$, and $\mathrm{Ni}_{3} \mathrm{Al}$. Some attempts to find superconductivity for the latter two compounds have failed up to now. Our recent theoretical results suggest that the insertion of boron into cubic $\mathrm{InSc}_{3}$ leads to superconductivity in $\mathrm{InSc}_{3} \mathrm{~B}$ compound [29]. In the paper [1] we observed in $\mathrm{Y}_{4} \mathrm{Co}_{3}$ the suppression of ferromagnetism by the onset of superconductivity at a lower temperature (the inverse of behaviour shown in majority of ferromagnetic superconductors), and the coexistence of both phenomena below $T_{\mathrm{S}}$. An examining of the theoretical possibility that weak itinerant ferromagnetism might be particularly amenable to such a take-over bid from superconductivity and of the coexistence state is a challenge up to now.

The band structure of $\mathrm{Y}_{9} \mathrm{Co}_{7}$ was measured by X-ray photoemission spectroscopy (XPS) in our paper from 1988 [30] but the spectrum was not of a high quality at that time and the comparison with band structure calculations [31] was not as well. In the paper [10] a speculated valence band structure of $\mathrm{Y}_{9} \mathrm{Co}_{7}$ was proposed, which may be responsible for the VWIF and also for superconductivity. That is why in the present paper we focus attention on our recent ultraviolet photoemission spectroscopy (UPS) of band structure of $\mathrm{Y}_{9} \mathrm{Co}_{7}$ and their comparison with our band structure calculations both of much better quality than that from nearly 20 years ago [30, 31].

In view of these facts it is a pity that almost no contemporary articles and reviews on the ferromagnetic superconductors have cited our papers on the first archetypical example of the weak itinerant ferromagnetic superconductivity and the coexistence in $\mathrm{Y}_{9} \mathrm{Co}_{7}$ even though the 25-years anniversary of this discovery has been already passed. That is why an equally important goal of the present paper is to remind to international audience that problem of coexistence of superconductivity and itinerant ferromagnetism started in fact 25 years ago since $\mathrm{Y}_{4} \mathrm{Co}_{3}[1]$. 


\section{Overview of previous experimental results}

All samples were usually produced by arc melting and then annealed at $850 \mathrm{~K}$ for two weeks and at $750 \mathrm{~K}$ for about six weeks. This heat treatment is very important because after such preparation procedure a specimen showed only one phase in X-ray diffraction and metallographic examinations. In high-purity samples even as high as $T_{\mathrm{S}} \approx 3.0 \mathrm{~K}$ might be reached [14]. The crystal structure of $\mathrm{Y}_{9} \mathrm{Co}_{7}\left(\mathrm{Y}_{4} \mathrm{Co}_{3}\right)$ was first determined as a so-called hexagonal $\mathrm{Ho}_{4} \mathrm{Co}_{3}$-type structure with the lattice parameters $a=11.53 \AA$ and $c=4.05 \AA$ [23]. Later, the structure was corrected to $\mathrm{Ho}_{4} \mathrm{Co}_{3+x}$-type structure [32], P63/m, space group 176. Finally, the structure was established as showed in Fig. 1, with the unit cell enlarged three times in comparison to that of $\mathrm{Y}_{4} \mathrm{Co}_{3}[2,14,17,33]$. Thus, the $\mathrm{Y}_{9} \mathrm{Co}_{7}$ compound can be regarded as a special type of solid solution $\mathrm{Y}_{12} \mathrm{Co}_{8}$ $\left(\square_{2-x} \mathrm{Co}_{x}\right)$ where $x=1.33$ and the symbol $\square$ represents the two special sites per unit cell of which 1.33 positions are occupied by Co.

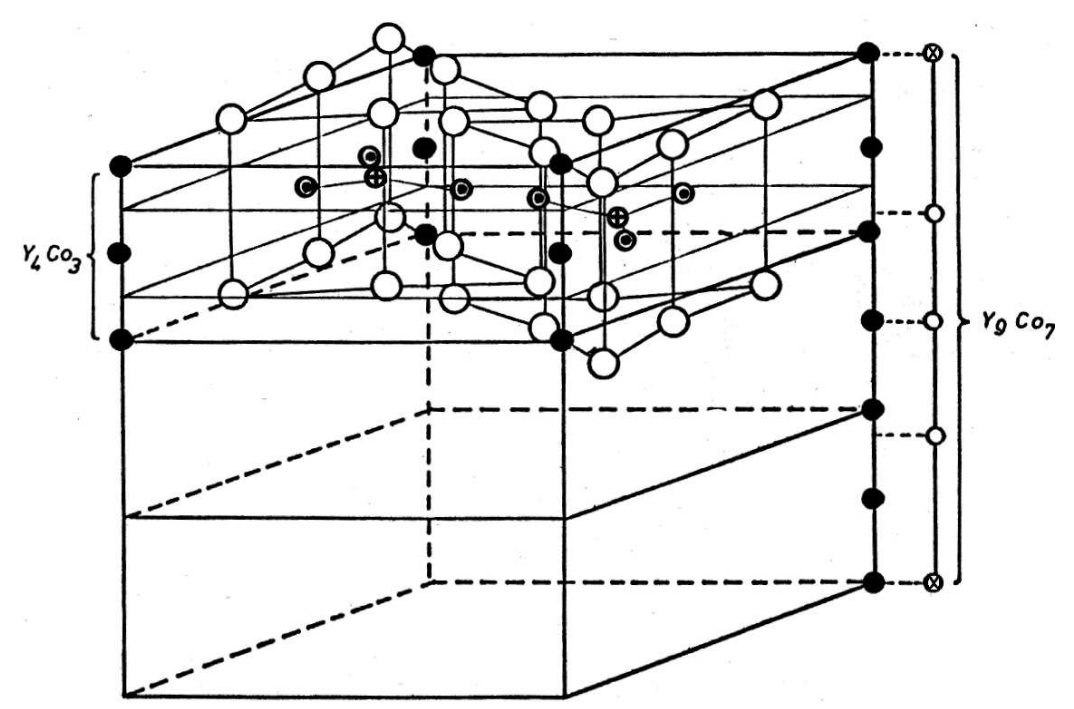

Fig. 1. The crystal structure of $\mathrm{Y}_{4} \mathrm{Co}_{3}$ as proposed in [32] and of $\mathrm{Y}_{9} \mathrm{Co}_{7}$ as proposed in $[2,33,42]$, where - is the b type site of $\mathrm{Co}, \oplus$ is the $\mathrm{d}$ type site of $\mathrm{Co}, \odot$ is the $\mathrm{h}$ type site of $\mathrm{Co}$ in $\mathrm{Y}_{4} \mathrm{Co}_{3}$, $\circ$ is the $\mathrm{b}$ type site of $\mathrm{Co}$ in $\mathrm{Y}_{9} \mathrm{Co}_{7}, \otimes$ is the isolated b type site of $\mathrm{Co}$ in $\mathrm{Y}_{9} \mathrm{Co}_{7}$, and $\mathrm{O}$ are the triangular prisms of the $\mathrm{Y}$ atoms.

For high quality specimens the X-ray and neutron diffraction patterns showed a single and stoichiometric phase of $\mathrm{Y}_{9} \mathrm{Co}_{7}$ with only a very small amount (less than 1 at.\%) of oxides $\mathrm{Y}_{2} \mathrm{O}_{3}$ and $\mathrm{Y}_{8} \mathrm{O}_{5}$ which are non-magnetic and non-superconducting compounds $[1,2,17,33]$.

The temperature dependences of the resistivity, magnetization and ac susceptibility were measured to reveal the most characteristic features of $\mathrm{Y}_{9} \mathrm{Co}_{7}$. The typical plots are shown in Fig. 2. 

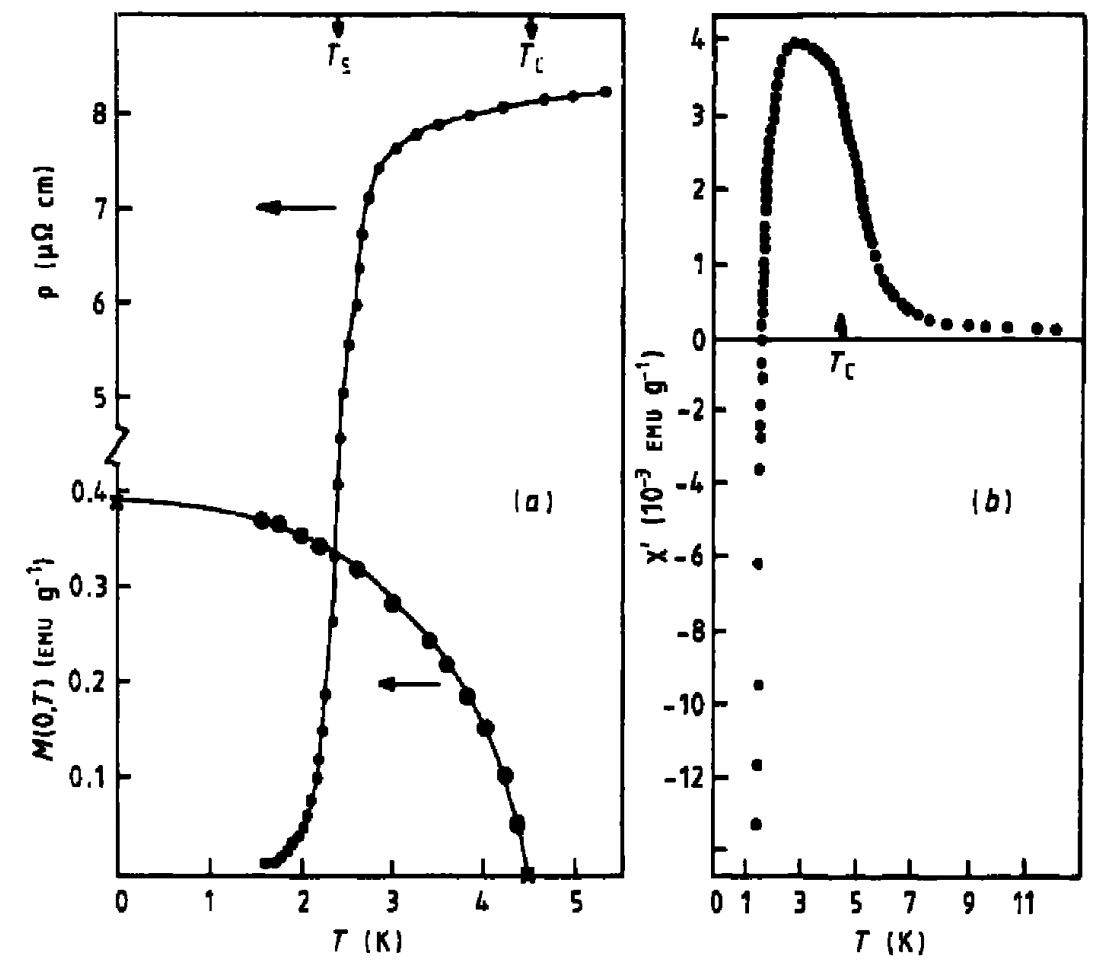

Fig. 2. The temperature dependences of resistivity $\rho$, the zero-field magnetization $M(0, T)$ (a) and the dynamic susceptibility $\chi^{\prime}$ of $\mathrm{Y}_{9} \mathrm{Co}_{7}$ (b) [11]. The zero-field magnetization is the extrapolated value for $H \rightarrow 0$ of the applied magnetic fields.

The resistance was usually measured by the standard dc or ac four-probe technique. The transition temperature to a superconducting state for the sample is $T_{\mathrm{S}}=2.4 \mathrm{~K}$ as the temperature at which the resistivity is half of that in the normal state (Fig. 1) or eventually as measured from an extrapolation of the upper critical field to zero field [5]. $\mathrm{Y}_{9} \mathrm{Co}_{7}$ was classified as a conventional superconductor with the Ginzburg-Landau-Abrikosov-Gorkov (GLAG) coherence length of about $315 \AA$, the GLAG penetration depth of about $1750 \AA$, the lower critical field of 92 Oe, the upper critical field of 3800 Oe, the superconducting energy gap of $0.7 \mathrm{meV}$ (all values extrapolated to zero temperature), and the effective electron-phonon coupling parameter $0.25[3,5,6,12,13]$. The superconducting properties are sensitive to the heat treatment during sample preparation whereas the magnetic properties are affected to a lesser extent. The residual resistance ratio $\mathrm{RRR}=R_{300 \mathrm{~K}} / R_{4.2 \mathrm{~K}}$ of the specimen was about 10 . A linear dependence was observed between the resistance ratio and the superconducting transition temperature, i.e. the higher RRR the higher $T_{\mathrm{S}}$ and the specimens with RRR as large as 35 were prepared $[2,3]$. Unfortunately, any good single crystal was manufactured up to now even though a number of attempts were carried out all over the world. 
The susceptibility measurements were usually undertaken in a small amplitude ac magnetic fields with the aim of throwing a more light on the character of the Meissner and critical state as well as of magnetic ordering. The in-phase ac susceptibility measurements were carried out using a conventional mutual inductance bridge with the amplitude of the ac magnetic field 0.1 Oe and an operating frequency of $323 \mathrm{~Hz}$, for a needle-like sample [1]. They show the diamagnetic superconducting behaviour below $1.7 \mathrm{~K}$, a rounded maximum at $T_{\mathrm{C}} \approx 4.5 \mathrm{~K}$ (see Fig. 2), and the modified Curie-Weiss law above $2 T_{\mathrm{C}}$ (see Fig. 1 in [10]):

$$
\chi(T)=\chi_{0}+\frac{C}{T-\theta}
$$

with $\chi_{0}=(2.25 \pm 0.01) \times 10^{-6} \mathrm{~cm}^{3} \mathrm{~g}^{-1}$, the Curie constant $C=$ $(2.29 \pm 0.01) \times 10^{-4} \mathrm{~cm}^{3} \mathrm{~g}^{-1} \mathrm{~K}$ and the paramagnetic Curie-Weiss temperature $\theta=(13.6 \pm 0.05) \mathrm{K}$. The effective magnetic moment $p_{\mathrm{C}}=(0.14 \pm 0.01) \mu_{\mathrm{B}} /$ Co atom was obtained from the expression for the Curie constant [10].

Below $2 \mathrm{~K}$ the ac susceptibility felt rapidly to negative values and reached the superconducting state at $T_{\mathrm{S}}^{*}=1.7 \pm 0.1 \mathrm{~K}$ as a crossing temperature point at which the susceptibility is zero. The susceptibility $\chi^{\prime}$ shows a clear diamagnetic response in the superconducting state, although the response was less than for an ideal Meissner state. However, later on for many very pure samples the complete Meissner state was reached. For example, the electrical resistivity and susceptibility measurements were made down to $0.35 \mathrm{~K}$ with the $\mathrm{He}^{3}$ cryostat $[1,14,19]$ and they showed the full Meissner state below about $1.0 \mathrm{~K}$ with diamagnetic response $\chi^{\prime} \approx-1.5 \times 10^{-2} \mathrm{emu} \mathrm{g}^{-1}$; the value very close to the theoretical value $\chi^{\prime}=-1 /(4 \pi \rho)$, where $\rho=5.85 \mathrm{~g} \mathrm{~cm}^{-3}$, for the perfect Meissner state.

The magnetization was measured as a function of the temperature from $4.365 \mathrm{~K}$ to $1.57 \mathrm{~K}$ and the magnetic field from -2.5 Oe to $-6.0 \mathrm{kOe}$ using a superconducting coil by a standard ballistic method [11]. The extrapolated zero-field magnetization values $M(0, T)$ for the magnetic isotherms [11]:

$$
\lim _{H \rightarrow 0} M(H, T)=M(0, T)=\left[\left(T_{\mathrm{C}}-T\right) / T^{*}\right]^{\beta},
$$

with the self-consistently fitted exponent $\beta=0.48 \pm 0.01$, evaluated from the proper analysis of the Arrot plots, are shown in Fig. 2. The details are presented in the paper [11]. The $M(0, T)$ obeys a quadratic behaviour in accordance with the spin fluctuation model of band weak ferromagnetism [11, 34, 35]. From the extrapolated zero-field zero-temperature magnetization $M(0,0)=0.383$ emu $\mathrm{g}^{-1}$ (see Fig. 2), the saturation magnetic moment was calculated to be $\mu_{\mathrm{S}}(0)=0.012 /$ Co atom. Taking into account the moment residing only on Co $2 \mathrm{~b}$ site in accord to the NMR results (cf. next Section), we have a saturation moment $\mu_{\mathrm{S}}(0) \approx 0.012 / \mathrm{Co}(\mathrm{b})$ atom. This value is the smallest one among all VWI ferromagnets known up to now. It was also established that the spin fluctuations play a large role in the temperature dependences of the resistivity, susceptibility above and below $T_{\mathrm{C}}[4,10-13]$ and in other properties. 
From these data one can conclude that VWIF and superconductivity coexist in $\mathrm{Y}_{9} \mathrm{Co}_{7}$ within some temperature interval below $T_{\mathrm{S}}$, say $\Delta T=1-5 \mathrm{~K}$. There was belief that such coexistence of superconductivity and itinerant ferromagnetism could not exist up to zero temperature. It seemed conceivable that the former could replace the latter at low temperatures. A piece of evidence on that problem came from the measurements of pressure dependence of ac susceptibility $[14,15]$. They showed that pressure suppresses the VWI ferromagnetism (cf. Fig. 2 in [15]), yielding the higher value of $T_{\mathrm{S}} \approx 3.5 \mathrm{~K}$ [14]. Moreover, from the results it was concluded that $\mathrm{Y}_{9} \mathrm{Co}_{7}$ is an itinerant ferromagnet, not a spin glass system, because the latter is rather insensitive to pressure [10-15]. Nevertheless, further experimental and theoretical examination whether very weak ferromagnetism might or might not be suppressed by superconductivity in $\mathrm{Y}_{9} \mathrm{Co}_{7}$ still has to be performed.

Many of differently prepared polycrystalline samples were examined all over the world by a number of experimental techniques besides the standard magnetic and transport methods. Among these techniques the specific heat $[9,14,18]$, the magnetoresistance [5], the pressure dependence of ac susceptibility $[14,15,18]$, the nuclear magnetic resonance (NMR) [9, 21, 36, 37], the X-ray and neutron diffraction structure determination [2, 33], the neutron depolarization [14], the muon spin relaxation [38], the electron tunelling spectroscopy [6], the Mössbauer spectroscopy [39], and the photoemission spectroscopy [31] were the most important in view to prove the coexistence of spin fluctuating VWIF with superconductivity, on microscopic scale in one unit cell of $\mathrm{Y}_{9} \mathrm{Co}_{7}$. In the last paragraph of this section we will concentrate only on some selected results of specific heat, magnetoresistance and NMR results. Next, we will present our new results on photoemission spectroscopy.

The specific heat at low temperatures was measured by the semi-adiabatic heat-pulse method $[9,14,18,22]$. It is shown in Fig. 3 [9] together with the temperature and magnetic field of magnetoresistance [5].

The relatively large temperature width of the specific heat transition to the superconducting state and to the magnetic state as well is most likely due to a magnetic field caused by the ferromagnetic domains of the sample and by inhomogeneous composition of the material but clear evidences of magnetic transition at $T_{\mathrm{C}} \approx 4.5 \mathrm{~K}$ and the superconducting transition at $T_{\mathrm{S}} \approx 2.0 \mathrm{~K}$ were detected. The electronic specific heat coefficient $\gamma=(3.4 \pm 0.1) \mathrm{mJ} \mathrm{mol}^{-1} \mathrm{~K}^{-2}$ (close to the value for metallic Co), and the Debye temperature $\theta_{\mathrm{D}}=(215 \pm 5) \mathrm{K}$ (close to that for yttrium), were calculated from the data [9].

The important discovery that there are three non-equivalent magnetic cobalt sites in the unit cell of $\mathrm{Y}_{9} \mathrm{Co}_{7}$ (cf. Fig. 1) was a result of NMR spin-echo measurements $[9,21,36,37]$ and also of the specific heat $[9,22]$ and the magnetoresistance measurements $[2,11,22]$. Two groups of NMR spin-echo lines were observed: the first exhibited a small but finite hyperfine field and the second had almost zero 


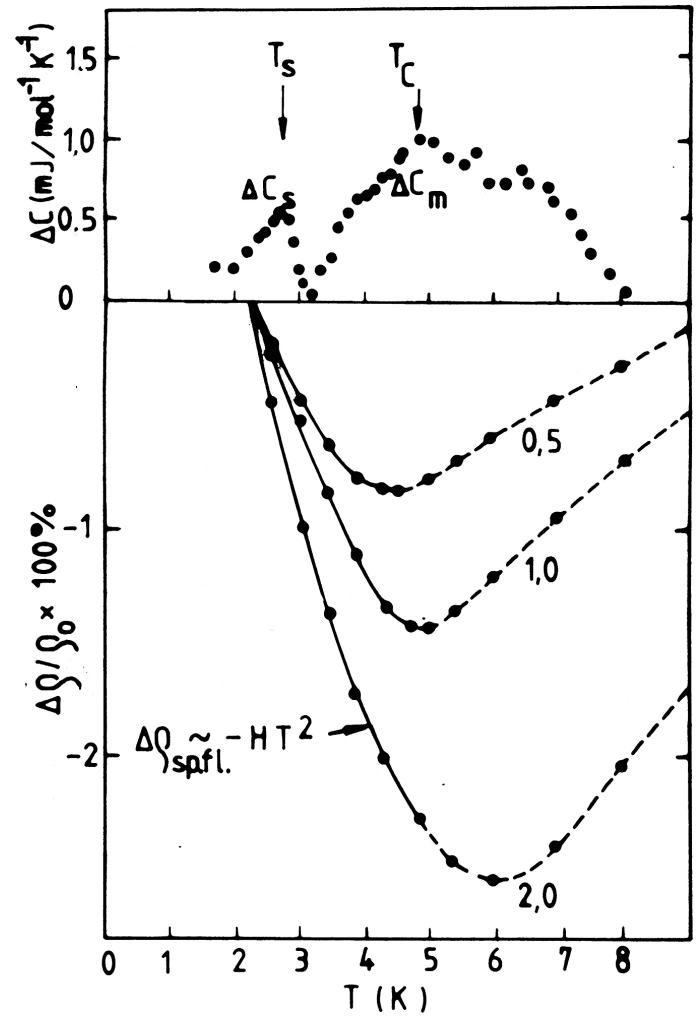

Fig. 3. The superconducting $\Delta C_{\mathrm{S}}$ and the magnetic $\Delta C_{\mathrm{m}}$ contributions to the specific heat versus temperature $[4,9]$ (upper part) together with the temperature dependence of magnetoresistivity (lower part), fitted to the self-consistent spin fluctuation (SCSF) [35] (solid line) in the given magnetic field.

hyperfine field. Thus, two different types of Co magnetic behaviour were suggested as a result of the three non-equivalent Co positions. From the NMR measurements it was established that the itinerant-electron ferromagnetism in the compound is of double character, consisting of a larger magnetic moment on the b-type Co atoms $\left(\approx 0.1 \mu_{\mathrm{B}} / \mathrm{Co}(\mathrm{b})\right.$ atom $)$, with some more local behaviour, and a very small itinerant magnetic moment on the d-type and h-type Co atoms. Both types of magnetic behaviour were related to the structure, the part of $\mathrm{Y}_{12} \mathrm{Co}_{8} \square_{2}$ would be a non-magnetic superconductor and the $\mathrm{Co}_{x}$ b-type atoms would carry the larger moments [1, 10-13].

As one can see from the above information a number of experiments were performed (for a review see $[4,12,14]$ ), but a more detailed piece of information on the origin of magnetism and superconductivity in $\mathrm{Y}_{9} \mathrm{Co}_{7}$ is needed especially from the electronic band structure point of view. 


\section{Photoemission results and their comparison with theoretical calculations \\ 3.1. Experimental}

In our previous paper on XPS experiments the core levels and valence bands of $\mathrm{Y}_{9} \mathrm{Co}_{7}$ and pure $\mathrm{Y}$ and Co were performed with an HP5950A spectrometer with monochromatized $\mathrm{Al} K_{\alpha}$ radiation $(h \nu=1387 \mathrm{eV})$ at room temperature. The energy resolution of kinetic energy of outcoming photoelectrons was $\Delta E \approx 0.7 \mathrm{eV}$ and the pressure during the measurements was $10^{-8}[30]$. Therein, the spectra were shown and the valence band spectrum was compared with the band-structure calculations of the local and total density of states for a given site and element [31]. The spectra and the comparison were not of a good quality. A conclusion was that the density of states at the Fermi level is large enough to give rise to the very weak itinerant ferromagnetism but the value is too small compared with the value calculated from the specific heat data $[5,9]$. In the paper [10] it was suggested that the properties of $\mathrm{Y}_{9} \mathrm{Co}_{7}$ are determined by a hybridized Co $3 d$ and $\mathrm{Y} 4 d$ band and by mutually interacting modes of magnetic spin fluctuations [10, 13].

In the present work the valence band (VB) spectra were detected at room temperature with the angle resolved ultraviolet photoemission spectrometer (ARUPS) equipped with the high energy resolution analyser AR 65 from Omicron, the Auger electron spectrometer (AES) and the low-energy electron diffractometer (LEED). The energy resolution of the spectrometer was $\Delta E \approx 70 \mathrm{meV}$, calibrated for the Fermi edge of $\mathrm{Ag}$, i.e. ten times better than in our previous work [30]. In fact, the UPS spectra were recorded for the angles $\theta=20^{\circ}$ of incident ultraviolet radiation and the angle $\phi=20^{\circ}$ of outcoming electrons with respect to the surface normal because we disposed of the polycrystalline samples and then the spectra did not depend on these angles. The high intensity ultraviolet helium source from FOCUS of energy $h \nu=21.2 \mathrm{eV}$ was used. The $\mathrm{Y}_{9} \mathrm{Co}_{7}$ plate-like shaped specimen of about $3 \times 6 \times 1.5 \mathrm{~mm}^{3}$ was cleaned in the preparation chamber by heating up to $500-700^{\circ} \mathrm{C}$, and annealed in situ in ultra-high vacuum conditions down to the lowest pressure in the $10^{-10}$ mbar range, until no surface contamination of oxygen and carbon could be detected by AES. Binding energies are related to the Fermi level $\left(E_{\mathrm{F}}=0 \mathrm{eV}\right)$ of a silver foil on which the samples were mounted.

\subsection{Electronic structure calculations}

Electronic structure calculations were performed using the fully self-consistent Korringa-Kohn-Rostoker Green function method with the coherent potential approximation (KKR-CPA) to treat chemical disorder [40, 41]. The crystal potential of muffin-tin form was constructed with the local density approximation (LDA) framework, using the von Barth and Hedin formula for the exchange-correlation part. For fully converged potentials (below $1 \mathrm{mRy}$ ), total, site-decomposed and $l$-decomposed density of states (DOS) were computed using tetrahedron technique in reciprocal space. The Fermi level was accurately determined from the generalized Lloyd formula [42]. 
In our calculations, a so-called hexagonal $\mathrm{Ho}_{4} \mathrm{Co}_{3}$-type structure (in fact $\mathrm{Ho}_{4} \mathrm{Co}_{3+x}$-type structure [32], P63/m, space group 176) was assumed (Fig. 1), which seems to be the most plausible one, according to neutron and X-ray diffraction studies $[17,33]$. In the $\mathrm{Ho}_{4} \mathrm{Co}_{3+x}$-type unit cell, yttrium atoms occupy 2 types of $6 \mathrm{~h}$ sites, whereas cobalt atoms are located on $6 \mathrm{~h}, 2 \mathrm{~d}$ and partially filled $2 \mathrm{~b}$ sites. The occupancy of $2 \mathrm{~b}$ site is about 1.333. Within the $\mathrm{Ho}_{4} \mathrm{Co}_{3+x}$-type structure the entitled " $\mathrm{Y}_{9} \mathrm{Co}_{7}$ " system corresponds to the disordered $\mathrm{Y}_{12} \mathrm{Co}_{9.33}$ alloy. The experimental lattice parameters $a=11.53 \AA, c=4.05 \AA$ and the atomic positions listed in Table were used in our computations. The disorder on $2 \mathrm{~b}$ site was represented by random distribution of a Co atom and a vacancy with filling of 1.33 and 0.67 , respectively.

TABLE

Atomic positions of $\mathrm{Y}_{9} \mathrm{Co}_{7}$ after [33] used in the calculations.

\begin{tabular}{c|c|c|c|c}
\hline \hline Site & $x$ & $y$ & $z$ & Occupation \\
\hline Y(I),6h & 0.7543 & 0.9791 & 0.7500 & 6 \\
& 0.0209 & 0.7752 & 0.7500 & \\
& 0.2248 & 0.2457 & 0.7500 & \\
& 0.2457 & 0.0209 & 0.2500 & \\
& 0.9791 & 0.2248 & 0.2500 & \\
\hline $\mathrm{Y}(\mathrm{II}), 6 \mathrm{~h}$ & 0.7752 & 0.7543 & 0.2500 & \\
& 0.1360 & 0.5150 & 0.7500 & 6 \\
& 0.4850 & 0.6210 & 0.7500 & \\
& 0.3790 & 0.8640 & 0.7500 & \\
& 0.8640 & 0.4850 & 0.2500 & \\
\hline $\mathrm{Co}(\mathrm{I}), 6 \mathrm{~h}$ & 0.5150 & 0.3790 & 0.2500 & \\
& 0.6210 & 0.1360 & 0.2500 & \\
& 0.4415 & 0.1578 & 0.7500 & 6 \\
& 0.7163 & 0.2837 & 0.7500 & \\
& 0.5585 & 0.8422 & 0.2500 & \\
\hline $\mathrm{Co}(\mathrm{III})$ & 0.1578 & 0.7163 & 0.2500 & \\
& 0.2837 & 0.4415 & 0.2500 & \\
& 0.6667 & 0.3333 & 0.7500 & 2 \\
& 0.3333 & 0.6667 & 0.2500 & \\
\hline & 0.0000 & 0.0000 & 0.0000 & \\
& & & &
\end{tabular}




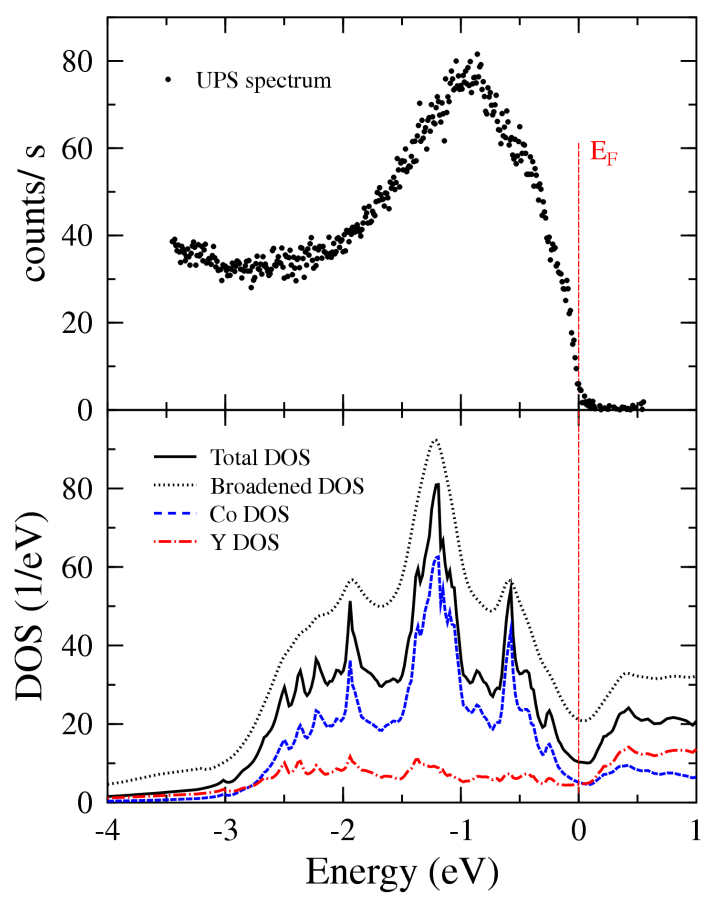

Fig. 4. UPS spectrum (upper part) and DOS of $\mathrm{Y}_{9} \mathrm{Co}_{7}$ (lower part) with the indicated atomic contributions. The total DOS broadened with the energy resolution $50 \mathrm{meV}$ is shown by the dotted most upper line.

Figure 4 presents the total density of states of " $\mathrm{Y}_{9} \mathrm{Co}_{7}$ " compound together with the experimental UPS spectrum of $\mathrm{Y}_{9} \mathrm{Co}_{7}$.

As one can see, the overall shape of the DOS curve and the UPS spectrum are quite similar, especially for the energy range of $-2<E<0 \mathrm{eV}$. At the first glance, the DOS consists of three main peaks, which can be attributed basically to Co $3 d$ states and the yttrium Y states contribution is much lower. Nevertheless, Y $4 d$ states become more pronounced for a higher energy and the DOS values are comparable to those on Co sites in the vicinity of the Fermi level $\left(E_{\mathrm{F}}\right)$. In the whole spectrum, one observes a strong hybridization of $d$-states of transition elements, which is partly responsible for a broad DOS minimum appearing near $E_{\mathrm{F}}$. Interestingly, comparing atomic DOS contributions at $E_{\mathrm{F}}$, we may notice that Co atoms located on the disordered $2 \mathrm{~b}$ site exhibit an important value of DOS at $E_{\mathrm{F}}$, being about three times larger than the corresponding contributions on $\mathrm{Co}(6 \mathrm{~h})$ and $\mathrm{Co}(2 \mathrm{~d})$ sites. Hence, the only non-vanishing local magnetic moment can be expected on $\mathrm{Co}(2 \mathrm{~b})$ site.

This conclusion remains in qualitative agreement with the experimental findings, which showed a very small magnetic moment on the Co $2 \mathrm{~b}$ site $\left(\approx 0.1 \mu_{\mathrm{B}}\right)$, as detected from NMR measurements [9, 21, 36, 37]. Let us note that it is rather 
difficult to stabilize such a small moment within the LDA calculations and more careful analysis is required. In order to enlighten superconducting and weakly ferromagnetic properties of $\mathrm{Y}_{9} \mathrm{Co}_{7}$ system, further electronic structure computations focused on critical parameters have been undertaken.

Our calculations differ from those recently published [43]. Therein, the crystal structure and DOS (Fig. 1 and Fig. 3 in [43]) would suggest that the author did not take into account the effect of disorder and partial filling of the 2b-Co site. Thus, the presented electronic structure corresponds to the hypothetical perfect $\mathrm{Y}_{12} \mathrm{Co}_{10}$ system, that is $\mathrm{Y}_{9} \mathrm{Co}_{7.5}$ against to the proper composition $\mathrm{Y}_{9} \mathrm{Co}_{7}$. In view of our results, the role of vacancy seems to be crucial for the electronic structure in the vicinity of the Fermi level.

\section{Conclusions}

1. $\mathrm{Y}_{9} \mathrm{Co}_{7}$ was classified as the typical conventional Bardeen-Cooper-Schrieffer (BCS) superconductor with a rather weak electron-phonon interaction, which can be properly described by the GLAG theory [3, 5, 6, 12, 13].

2. The temperature and magnetic field dependences of resistivity, susceptibility, magnetization, specific heat and of other properties (Figs. 2 and 3) in the ferro- and paramagnetic region were consistently described and understood taking into account a significant effect of spin fluctuation [10-13] within the framework of SCSF theory [35].

3. From the enhancement of superconductivity due to the suppression of ferromagnetism under pressure in $\mathrm{Y}_{9} \mathrm{Co}_{7}[14,15,18]$ and from other experiments $[9,10-14,18,38]$ a few evidences were found that the ferromagnetism is very weak and itinerant (VWIF), but not a spin glass-like ordering, as well as that both VWIF and superconductivity are strongly linked forming a coexistence state.

4. From the NMR $[9,21,36,37]$ and the present photoemission investigations, the conclusion was drawn that the weak itinerant-electron ferromagnetism in the compound is of double character, consisting of a larger magnetic moment on the b-type Co atoms $\left(\approx 0.1 \mu_{\mathrm{B}} / \mathrm{Co}(\mathrm{b})\right.$ atom $)$, with some more local behaviour and a very small itinerant magnetic moment on the d-type and h-type Co atoms.

5. The comparison of the present photoemission valence band spectra with the calculated shape of the DOS curve (cf. Fig. 4) exhibits a very good similarity. The Co atoms located on the disordered $2 \mathrm{~b}$ site participate significantly to the value of DOS at $E_{\mathrm{F}}$, being about three times larger than the corresponding contributions on $\mathrm{Co}(6 \mathrm{~h})$ and $\mathrm{Co}(2 \mathrm{~d})$ site. $\mathrm{Y} 4 d$ states become more pronounced for a higher energy. There exists a strong hybridization of $3 d-$ and $4 d$-states in $\mathrm{Y}_{9} \mathrm{Co}_{7}$. 
6. From all these data one can conclude that $\mathrm{Y}_{9} \mathrm{Co}_{7}$ is a very weak itinerant ferromagnetic superconductor and a first archetypical example of the coexistence of both phenomena in some temperature interval below $T_{\mathrm{S}}$.

\section{Acknowledgments}

This paper is dedicated to Professor Karol Krop on the occasion of his 70th birthday anniversary and to Professor Józef Spałek on his 60th birthday anniversary. One of us (A.K.) thanks a lot to both Professors for their permanent interest and help in $\mathrm{Y}_{9} \mathrm{Co}_{7}$ problem. This work was financially supported by the Faculty of Physics and Applied Computer Science, AGH University of Science and Technology, Cracow, and from the Ministry of Science and Higher Education, Poland.

\section{References}

[1] A. Kołodziejczyk, B.V.B. Sarkissian, B.R. Coles, J. Phys. F, Met. Phys. 10, L333 (1980).

[2] A.K. Grover, B.R. Coles, B.V.B. Sarkissian, H.E.N. Stone, J. Less-Common Met. 86, 29 (1982); B.V.B. Sarkissian, A.K. Grover, J. Phys. F, Met. Phys. 12, L107 (1982); V.B. Sarkissian, A.K. Grover, B.R. Coles, Physica B 109-110, 2041 (1982).

[3] C. Sułkowski, K. Rogacki, A. Kołodziejczyk, G. Kozłowski, J. Chmist, J. Phys. F, Met. Phys. 13, 2147 (1983).

[4] A. Kołodziejczyk, Physica B 130, 189 (1985) and references cited therein.

[5] A. Kołodziejczyk, C. Sułkowski, in: Proc. Low Temperature Conference LT-17, Karlsruhe (Germany) 1984, Eds. U. Eckern, A. Schmid, W. Weber, H. Wull, Elsevier Sci. Publ., Amsterdam 1984, p. BN 14.

[6] A. Kołodziejczyk, J. Raułuszkiewicz, A. Reich, B.V.B. Sarkissian, Acta Phys. Pol. A 68, 133 (1985).

[7] E. Gratz, J.O. Strom-Olsen, M.J. Zukerman, Solid State Commun. 40, 833 (1981).

[8] Y. Yamaguchi, Y. Nishihara, S. Ogawa, J. Magn. Magn. Mater. 31-34, 513 (1983).

[9] A. Lewicki, Z. Tarnawski, A. Kołodziejczyk, Cz. Kapusta, H. Figiel, J. Chmist, Z. Lalowicz, L. Śniadower, J. Magn. Magn. Mater. 36, 291 (1983).

[10] A. Kołodziejczyk, J. Spałek, J. Phys. F, Met. Phys. 14, 1277 (1984).

[11] A. Kołodziejczyk, C. Sułkowski, J. Phys. F, Met. Phys. 15, 1151 (1985).

[12] A. Kołodziejczyk, Sci. Bull. Acad. Mining Metall. no. 1041, ser. Phys. 2, 1-92 (1986), habilitation in English, and references cited therein.

[13] A. Kołodziejczyk, J. Magn. Magn. Mater. 70, 8 (1987).

[14] B.V.B. Sarkissian, J. Appl. Phys. 53, 8071 (1982) and references cited therein; B.V.B. Sarkissian, J. Beille, J. Appl. Phys. 54, 2004 (1984).

[15] C.Y. Huang, C.E. Olsen, W.W. Fuler, J.H. Huang, S.A. Wolf, Solid State Commun. 45, 795 (1983).

[16] Y. Yamaguchi, Y. Nishihara, S. Ogawa, J. Phys. Soc. Jpn. 53, 3985 (1984). 
[17] K. Yvon, F.H. Braun, E. Gratz, J. Phys. F, Met. Phys. 13, L135 (1983).

[18] A. van der Liet, P.H. Frings, A. Menovsky, J.J.M. Franse, J.A. Mydosh, G.J. Nieuwenhuys, J. Phys. F, Met. Phys. 12, L153 (1982).

[19] J. Sebek, J. Stehno, V. Sechovsky, E. Gratz, Solid State Commun. 40, 457 (1981).

[20] E. Gratz, R. Kirchmayr, V. Sechovsky, E.P. Wohlfarth, J. Magn. Magn. Mater. 21, 191 (1980).

[21] H. Figiel, E. Gratz, Cz. Kapusta, J. Magn. Magn. Mater. 23, 123 (1981).

[22] W. Cheng, G. Creuzet, P. Garoche, I.A. Campbell, E. Gratz, J. Phys. F, Met. Phys. 12, 475 (1982).

[23] C. Berthet-Colominas, J. Laforest, R. Lemaire, R. Pauthenet, J. Schweizer, Cobalt 39, 97 (1968).

[24] Ø. Fischer, Magnetic Superconductors - Ferromagnetic Materials, Eds. K.H.J. Bushow, E.P. Wolfarth, Elsevier, Amsterdam 1990, p. 467; B. Maple, Ø. Fisher, Topics in Current Physics 34, 1 (1982).

[25] J. Flouquet, A. Huxley, D. Braithwaite, F. Hardy, G. Knebel, V. Mineev, E. Ressouche, D. Aoki, J.P. Brison, Acta Phys. Pol. B 34, 275 (2003) and references cited therein.

[26] S.S. Saxena, P. Agarwal, K. Ahilan, F.M. Grosche, R.K.W. Haselwimmer, M.J. Steiner, E. Pugh, I.R. Walker, S.R. Julian, P. Monthoux, G.G. Lonzarich, A. Huxley, I. Sheikin, D. Brathwaite, J. Flouquet, Nature 406, 587 (2000); S.S. Saxena, P.B. Littlewood, Nature 412, 290 (2001).

[27] C. Pfleiderer, M. Uhlarz, S.M. Hayden, R. Vollmer, H. v. Löhneysen, N.R. Bernhoeft, G.G. Lonzarich, Nature 412, 58 (2001).

[28] D. Aoki, A. Huxley, E. Ressouche, D. Braithwaite, J. Flouquet, J.-P. Brison, E. Lhotel, C. Paulsen, Nature 413, 613 (2001).

[29] B. Wiendlocha, J. Toboła, S. Kaprzyk, Phys. Rev. B 73, 134522 (2006); B. Wiendlocha, J. Tobola, S. Kaprzyk, D. Fruchart, J. Marcus, Phys. Status Solidi B 243, 351 (2006).

[30] P. Steiner, B. Siegwart, I. Sander, A. Kołodziejczyk, K. Krop, J. Phys. F, Met. Phys. 18, L 241 (1988).

[31] M. Shimizu, A. Kunihara, J. Inoue, J. Phys. F, Met. Phys. 16, 1263 (1986).

[32] R. Lemaire, J. Schweizer, J. Yakinthos, Acta Crystallogr. B 25, 710 (1969).

[33] A. Kołodziejczyk, J. Leciejewicz, A. Szytuła, J. Chmist, R. Wȩgrzyn, Acta Phys. Pol. A 72, 319 (1987).

[34] G.G. Lonzarich, L. Taillefer, J. Phys. C, Solid State Phys. 18, 4339 (1985).

[35] T. Moriya, J. Magn. Magn. Mater. 14, 1 (1979); T. Moriya, in: Electron Correlation and Magnetism in Narrow Band System, Ed. T. Moriya, Springer-Verlag, Berlin 1981, p. 2.

[36] M. Takigawa, H. Yasuoka, Y. Yamaguchi, S. Ogawa, J. Phys. Soc. Jpn. 52, 3318 (1983).

[37] S. Wada, T. Kohara, K. Asayama, Y. Kitaoka, Y. Kohori, N. Ishikawa, Solid State Commun. 48, 5 (1983).

[38] E.J. Ansaldo, D.R. Noakes, J.H. Brewer, R. Keitel, D.R. Harshman, M. Senba, C.Y. Huang, B.V.B. Sarkissian, Solid State Commun. 55, 193 (1985). 
[39] A. Kołodziejczyk, J. Żukrowski, J. Phys. F, Met. Phys. 15, L 217 (1985).

[40] A. Bansil, S. Kaprzyk, P.E. Mijnarends, J. Tobola, Phys. Rev. B 60, 13396 (1999).

[41] T. Stopa, S. Kaprzyk, J. Tobola, J. Phys., Condens. Matter 16, 4921 (2004).

[42] S. Kaprzyk, A. Bansil, Phys. Rev. B 42, 7358 (1990).

[43] T. Jeong, Solid State Commun. 138, 262 (2006). 